Revista Universo Contábil, ISSN 1809-3337

FURB, v. 5, n.4, p. 152-161, out./dez., 2009

doi:10.4270/ruc.2009436

Disponível em www.furb.br/universocontabil

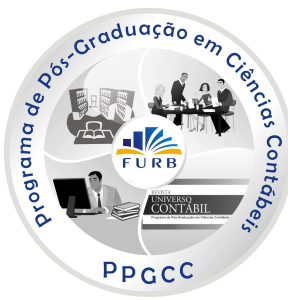

\title{
APLICAÇÃO DAS NORMAS CONTABILÍSTICAS INTERNACIONAIS IAS 32, IFRS 7 E IAS 39 EM PRÁTICA DAS ENTIDADES EMPRESARIAIS DA REPÚBLICA CHECA*
}

\section{APLICAÇÃO DAS NORMAS CONTÁBEIS INTERNACIONAIS IAS 32, IFRS 7 E IAS 39 NA PRÁTICA DAS ORGANIZAÇÕES EMPRESARIAIS DA REPÚBLICA CHECA}

\section{INTERNATIONAL STANDARDS FOR REPORTING OF FINANCIAL INSTRUMENTS (IAS 39, IFRS 7 AND IAS 32) IN THE COMMON PRACTICE OF CZECH COMPANIES}

\author{
Jiri Strouhal \\ Doutor em Contabilidade e Auditoria pela Universidade de Economía Praga \\ Senior Lecturer da Universidade de Economía Praga \\ Endereço: W. Churchill, Square 4 \\ CEP: 13067 - Praga - República Checa \\ E-mail: strouhal@vse.cz \\ Telefone: +420224 095-794
}

\section{RESUMO}

É um facto que, uma vez que as entidades reguladoras adoptam um determinado paradigma de informação financeira, este torna-se o princípio orientador para a regulamentação da contabilidade. Este paradigma que domina a contabilidade representa o ponto de partida deste trabalho de investigação, o qual incide sobre o caso específico da República Checa, mais concretamente nos aspectos relativos às regras que dizem respeito aos instrumentos financeiros. Ao nível da análise económica foi conduzido um trabalho de inferência, à priori das regulamentações nacionais em consonância com padrões internacionais. Ao analisar minuciosamente o normativo contabilístico respeitante aos instrumentos financeiros não foram identificadas questões-chave que precisam ser discutidas. Este artigo representa um primeiro passo no sentido de entender cientificamente, uma investigação à priori com a finalidade de gerar feedback sobre hipotéticas alternativas de relatórios antes de sua implementação. Adicionalmente, os temas analisados sob a perspectiva regulamentar devem ser particularmente avaliados em relação à sua aplicação efectiva, às conclusões pertinentes e correlações com o status do país no mercado global de capitais.

Palavras-chave: Informação financeira. Instrumentos financeiros. Harmonização. República Tcheca.

Artigo recebido em 18.09.2008. Revisado por pares em 04.11.2008. Reformulado em 20.01.2009. Recomendado em 28.01.2009 por Ilse Maria Beuren (Editora). Publicado em 30.11.2009. Organização responsável pelo periódico: FURB. 


\section{RESUMO}

É fato que, uma vez que as entidades reguladoras adotam um paradigma de informação financeira, este se torna o princípio orientador para a regulamentação da contabilidade. Este paradigma que domina a contabilidade representa o ponto de partida desta investigação, incidindo sobre o caso específico da República Tcheca, mais especificamente nos aspectos relativos às regras que dizem respeito aos instrumentos financeiros. Numa análise econômica, a priori das regulamentações nacionais em consonância com padrões internacionais, inferências foram realizadas. Não foram identificadas várias questões-chave que precisam ser discutidas ao analisar minuciosamente os regulamentos contábeis para instrumentos financeiros. Este artigo representa um primeiro passo na direção do entendimento científico, uma investigação a priori com a finalidade de gerar feedback sobre as alternativas hipotéticas de relatórios antes de sua implementação. Além disso, os temas analisados sob a perspectiva regulamentar devem ser particularmente avaliados referente à sua aplicação efetiva, às conclusões pertinentes e correlações com o status do país no mercado global de capitais.

Palavras-chave: Informação financeira. Instrumentos financeiros. Harmonização. República Tcheca.

\section{ABSTRACT}

It is well known the fact that once regulatory bodies adopt a financial reporting paradigm, it becomes the guiding principle for accounting regulation. This paradigm itself in the field of accounting represents the starting point of our research which focuses on the specific case of Czech Republic, namely on aspects concerning regulations in the field of financial instruments. An a priori economic analysis of national regulations in correlation with international standards inferences has been approached. In doing so, there were identified several key issues, which need to be discussed when thoroughly analyzing accounting regulations for financial instruments. Paper represents a first step within intended scientific demarche, a priori research having the quality of generating feedback on hypothetical reporting alternatives prior to implementation. Forward, those issues analyzed through the regulations perspective need to be closely quantified in matters of their actual implementation, pertinent conclusions and correlations being then made regarding the status of the country within the global capital market.

Keywords: Financial reporting. Financial instruments. Harmonization. Czech Republic.

\section{INTRODUÇÃO}

A partir do dia $1^{\circ}$. de maio de 2004, as entidades empresariais cujos títulos são negociados publicamente devem apresentar suas demonstrações financeiras conforme as normas internacionais para relatórios financeiros (IFRS). As normas mais discutidas em termos da sua implementação são as IAS 32 Instrumentos Financeiros: Apresentação; IFRS 7 Instrumentos Financeiros: Divulgação; e IAS 39 Instrumentos Financeiros: Reconhecimento e Mensuração.

A pesquisa no ramo da harmonização dos processos contabilísticos focalizava-se sobretudo em dois aspectos essenciais, ou seja segurança e exactidão da medição do valor, por exemplo Aisbitt (2001), Emenyonu e Grey (1992 a 1996), Herman e Thomas (1995). 
Segundo Alexandr e Nobes (2004) é possível definir os seguintes factores que influenciam o desenvolvimento das normas contabilísticas em respectivo país: influências coloniais e externas, influência dos provedores do capital, carácter do sistema nacional jurídico, influência dos impostos e taxas obrigatórias, influência da profissão de contabilistas.

Em 2002 o Conselho da União Europeia publicou o Regulamento através que obrigou as sociedades cujos títulos são negociados publicamente nas bolsas europeias respeitarem nas suas demonstrações financeiras consolidadas as normas de IFRS, o mais tardar a partir de 2005. Antes da aplicação das regras comuns existiram 27 métodos diferentes para as demonstrações financeiras das sociedades cujos títulos são negociáveis publicamente (WHITTINGTON, 2005, p. 129). Brown e Tarca (2005, p. 201) prevêem que o futuro da IASB depende, sobretudo, da boa implementação das IFRS na Europa.

Apesar da IFRS não representar uma alternativa equivalente para as demonstrações financeiras no nosso país, certas entidades empresariais devem respeita-las nos seus balanços anuais, conforme exigido pela Lei sobre Contabilidade. A excepção abrange as entidades contabilísticas consolidadas, que emitem títulos registados no mercado regulado dos títulos de valor nos países-membros da União Europeia. Outras entidades que elaboram demonstrações financeiras consolidadas podem escolher entre o balanço de contas conforme as normas nacionais e o balanço de contas conforme IFRS. Entre as maiores vantagens da aplicação das normas internacionais destacam: um acesso facilitado aos mercados estrangeiros, a credibilidade reforçada das sociedades estrangeiras no mercado nacional, a comparabilidade global dos dados contabilísticos, dados mais transparentes e mais compreensíveis graças ao „idioma comum de contas“, regulação facilitada nos mercados de capital, maior resistência das normas contabilísticas ás influências políticas.

Ainda antes de a UE ter adoptado as normas IFRS, as bolsas de valores exigiam que as entidades com títulos negociados publicamente apresentassem os balanços de contas conforme IFRS ou US GAAP. As pesquisas anteriores sobre o grau da publicidade (COOKE, 1992; MEEK et al., 1995), e da probabilidade da aplicação das normas supranacionais (ELGAZZAR et al., 1999; MURPHY, 1999; ASHBAUGH, 2001; DUMONTIER e RAFFOURNIER, 1998; LEUZ e VERRECCHIA, 2000; LEUZ, 2003) indicam uma correlação positiva entre a cotação das entidades contabilísticas nos mercados estrangeiros e o grau da publicidade e/ou aplicação das normas supranacionais como a base das demonstrações financeiras.

A principal contribuição da norma IAS 39 consiste em aplicação alargada do modelo do justo valor em medição do valor dos instrumentos financeiros. É verdade que os custos históricos costumavam servir da base de medição do valor, sendo aplicados também no ramo dos instrumentos financeiros. Nos últimos anos tinha crescido o significado e volume dos negócios de derivados, que, tendo sido aplicado neles o modelo dos valores históricos, acabaram com um valor de zero. Assim sublinha Whittington (2005), que é muito melhor valorizar os derivados na base dos preços correntes, o que corresponde ao justo valor definido pela norma IAS 39 .

Do outro lado, alguns peritos receiam que a aplicação da IAS 39 irá conduzir à maior volatilidade do resultado do exercício e do capital próprio, e que essa volatilidade não necessariamente corresponderá com a volatilidade económica real. Aliás, estudo anterior (por exemplo, Leftwich, 1981) indica que a aplicação das diferentes normas de contabilidade geralmente não coincide com as mudanças dos valores dos instrumentos financeiros caso na haver outras consequências económicas da adopção dessas regras.

Há também muitos estudos sobre a aplicação dos justos valores sobre as carteiras dos títulos de investimento dos bancos. Riffe (1993) e McAnally (1995), entre outros, analisaram a capacidade informativa da norma norte-americana SFAS 105 para as entidades bancárias. 
Riffe (1993) tinha provado a existência da interacção causativa entre os valores reconhecidos em sub-balanço de contas e o valor do capital próprio.

Outros estudos analisaram o emprego do justo valor em medição dos instrumentos financeiros. Barth et al. (1995) examinaram o efeito exercido pela avaliação na base do justo valor sobre a volatilidade do resultado de exercício e o preço das acções. Eles tinham provado que tanto o lucro como o valor do capital próprio baseado nas contas com justo valor mostra a volatilidade superior à das contas reconhecidas na base dos custos históricos, e que essa volatilidade adicional influencia significativamente o valor das acções.

Pirchegger (2006) receia que as entidades contabilísticas, em caso da contabilização das operações de hedge/cobertura, podem sobrestimar a importância do dever de informar, e os custos relacionados. Os autores das normas também tomaram por importante que os investidores tivessem à sua disposição o máximo das informações relevantes. $\mathrm{O}$ facto de ter incluído as informações da contabilização das operações de hedge entre os dados indispensáveis do balanço de contas era muito mais motivado pelo desejo de proporcionar para os investidores dados mais seguros do que pela aplicação correcta dos critérios válidos para a contabilização de hedge. Apesar do que as informações sobre operações de hedge causam muitos custos adicionais para as entidades contabilísticas.

A relação entre os conceitos económico e contabilísticos das operações de hedge tinha sido examinada por um grande número de estudo. Melumad et al. (1999) mostra que o reconhecimento da hedge/cobertura conforme a norma americana SFAS 133 resulta em desvios da hedge ideal, como essa é entendida pelas regras económicas. Ao mesmo tempo, Barnes (2001) avisa que esses desvios da hedge económica resultam justamente do modelo da contabilização das operações de hedge. Ele salienta que essa contabilização das operações de hedge pode motivar as empresas cujos resultados de exercício não estão bons para especularem, e também para influenciarem seus resultados de exercício em curto prazo.

Os efeitos informativos e controladores da contabilização das operações de hedge estado examinados por vários estudos (JORGENSEN, 1997; HUDHES et al., 2002). O mais interessante parece o facto que a aplicação voluntária da contabilização das operações de hedge/cobertura resulta em desvio da melhor estratégia de hedge (em contraste à situação quando a empresa realiza as operações de cobertura em termos económicos mas não aplica a regras da contabilização de hedge).

O reconhecimento dos títulos de valores realizáveis, ou eventualmente destinados para serem negociados, obedece às regras semelhantes tanto no ambiente nacional, como no ambiente da IFRS. Por consequência, o impacto sobre balanço de contas é idêntico tanto em caso de HFT como em caso de AFS. A diferença essencial consiste em significativamente maior exigência das informações por parte da norma IFRS.

A experiência do reconhecimento dos títulos de crédito (posição do investidor) indica que a maior diferença aparece em métodos de amortização das diferenças do valor nominal e o custo de aquisição do título (amortização de desconto ou prémios relacionados). No âmbito checo, as entidades preferem a distribuição linear dos custos ou dos proveitos (muitas vezes motivadas pela situação fiscal), a IFRS exige a amortização na base da taxa de juro efectiva. Esse facto causa as principais diferenças em resultados de exercício demonstradose valores contabilísticos dos títulos de crédito em cada ano.

\section{NOTAS CRÍTICAS}

Os instrumentos financeiros continuam inspirar emoções. O tratamento contabilístico do reconhecimento e demonstração dos instrumentos financeiros em termos do disposto nas normas internacionais associa-se, até certo ponto, com a questão do valor justo ou, mais propriamente, valor actual. Isso resulta do desejo dos autores, oferecer para os investidores 
que estão a preparar o balanço de contas os dados mais actualizados e recentes, com referência ao desenvolvimento recente do valor de mercado dos activos da respectiva empresa.

Trata-se, sem dúvida, de uma tentativa respeitável, mas a sua aplicabilidade depende sobretudo e essencialmente de eficácia e transparência do mercado. E até agora não parece que essas são as qualidades que caracterizem o mercado checo. Apesar de o justo valor ser um das bases de valorização na $\mathrm{RCh}$, seu cálculo baseia-se muitas vezes nos modelos de valorização, em vez de o preço de mercado.

Ao comparar as bases de valorização das IFRS e das práticas checas, não podemos esquecer de mencionar a impossibilidade de valorizar as dívidas e créditos de longo prazo na base do valor actual (ou custos amortizados), visto que as entidades do mercado checo devem reconhecer tanto as dívidas e créditos de curto prazo, como os de prazo superior a um ano, em valores nominais. Um desrespeito absoluto do valor actual do dinheiro pode exercer um impacto significativo sobre as demonstrações financeiras (apesar de obedecer as regras vigentes) e, eventualmente, prestar informações pouco adequadas para a decisão dos investidores.

\section{PROBLEMAS RELACIONADOS COM A VALORIZAÇÃO E RECONHECIMENTO DOS TÍTULOS DE VALOR}

\subsection{Acções}

No âmbito checo, as unidades contabilísticas devem revalorizar os títulos patrimoniais seja pelo método da equivalência patrimonial ou pelo justo valor, consoante o tipo de carteira/portfolio em que foram incluídos.

O valor de mercado do respectivo instrumento considera-se a melhor fonte da informação sobre o justo valor do instrumento, e quando impossível de identificar, a unidade utiliza a medição pericial realizada na base do modelo de medição.

Infelizmente, o procedimento ideal na base do valor de mercado, falha por falta da transparência do mercado checo de acções, e aplicam-se geralmente outros modelos. As desvantagens da avaliação na base do valor líquido dos activos consistem na existência das várias bases utilizadas nos processos contabilísticos, e também o princípio de prudência muito preferido pelo mercado checo e impossibilidade de supra-valorizar certos tipos de património (ao contrário de IFRS).

Serve da boa informação sobre o justo valor sua derivação da relação P/E. Cumpre-se dizer que o modelo não presta caso a entidade tiver o resultado de exercício negativo, e ademais é um critério difícil de obter no âmbito do mercado checo.

A desvantagem principal do modelo de desconto de dividendos consiste no facto de a sua aplicação estar condicionada com o crescimento constante dos dividendos, muito raro na vida prática. Apesar disso, o modelo é aplicável para o cálculo do dividendo em dois períodos consequentes mas falha, caso a entidade não distribui os dividendos ou tem o resultado de exercício negativo.

No âmbito checo será sempre possível reconhecer o título de valor em custo de aquisição, caso não for possível reconhece-lo em valor justo. As entidades checas, nas suas demonstrações financeiras, muitas vezes não utilizam o método da equivalência patrimonial (aplica-se o direito de escolha) as suas participações nas empresas controladas e nas empresas onde a companhia não é detentora maioritária, mas reconhece as suas participações em custos de aquisição, eventualmente ajustados.

$\mathrm{Na}$ altura em que os custo de reavaliação dos títulos de participação eram reconhecidos como dedutíveis do imposto até o nível do rendimento da respectiva reavaliação, as entidades criticavam o sistema e preferiam a reavaliação de capital. Hoje em 
dia, a reavaliação dos títulos de valor de curto prazo não exerce impacto no cálculo de imposto e o problema deixou se existir.

\subsection{Títulos de dívida}

Não há discrepância material entre as normas nacionais e requisitos das normas internacionais de IFRS em caso do reconhecimento contabilístico dos títulos de dívida com o prazo de vencimento inferior a um ano. Mas devemos constatar que o reconhecimento contabilístico desses instrumentos financeiros em caso do prazo de vencimento superior a um ano não é consistente com a norma de IFRS. O custo de aquisição do investimento em obrigações está sujeito ao crescimento proporcionado pelo valor de desconto/prémio amortizado; do ponto de vista da entidade emitente os contratos são reconhecidos em valores nominais, em vez de valores actuais.

Assim não se pode comparar as demonstrações financeiras, a parte do reconhecimento do investimento de longo prazo em obrigações, das sociedades cotadas e não cotadas. As entidades contabilísticas do mercado checo podem preferir a aplicação do $\S 7$ da Lei sobre a contabilidade e do reconhecimento fiel e justo, mas ao mesmo tempo continuam achar importante o seu aspecto fiscal.

\subsection{Contractos de derivados}

O maior problema relacionado com o reconhecimento dos contractos de derivados nos livros das entidades empresariais consiste em mensuração do valor justo daqueles instrumentos financeiros, mas também dos valores justos dos activos e passivos. $\mathrm{O}$ valor justo é (também por definição) a quantidade pela qual um activo pode ser trocado ou um passivo pode ser vendido, entre duas partes independentes, conhecedoras do negócio e dispostas a finalizar a operação. Entretanto, nas negociações sobre os instrumentos financeiros de derivados existia e provavelmente continuará existindo uma assimetria de informações das quais dispõe a empresa, de um lado, e a sociedade, parte do contrato, do outro lado.

Uma absoluta maioridade das empresas não é capaz de medir os valores justos dos seus contratos de derivados e fica dependente das informações fornecidas pelas sociedades financeiras contratadas. As empresas muitas vezes acabam dispor de informações sobre os valores justos dos instrumentos, e já não dos valores justos dos activos e passivos de subbalanço, que resultam do respectivo contrato de derivados.

Nas versões adoptadas pela Alemanha e pela Áustria, o balanço de contas não reconhece os derivados. Considerando os contratos de derivados um negócio pouco seguro, essas versões requerem que o balanço reconheça-o somente em caso de perigo de perdas causadas por esses negócios. Do outro lado, as entidades contabilísticas devem incluir em comentário as informações sobre o tipo e quantidade dos derivados financeiros, seu valor justo e método de avaliação.

Em relação com os contratos de derivados convém mencionar o nível insuficiente das informações publicadas, falta da atitude unificada das empresas para com as informações, evidente não só nas demonstrações financeiras das várias entidades, mas também nas demonstrações financeira de uma empresa. Assim vira muito difícil comparar e quantificar as informações publicadas.

Geralmente há informações sobre a estrutura dos derivados, seus valores nominais e justos, tipos dos instrumentos, seu prazo, desenvolvimento, etc. Espalhadas nas páginas do balanço de contas. As informações podem ser mais ou menos detalhadas, as empresas reconhecem-nas em formas diferentes. Por exemplo, o valor nominal reconhece-se individualmente para cada tipo do instrumento de derivado, mas o valor justo é reconhecido de maneira acumulada, dependente do risco coberto pelo respectivo derivado. 
A maioria das entidades contabilísticas da República Checa declara que não utiliza os derivados para os fins especulativos. Devido à escassez das informações sobre os derivados existe, aliás, o risco de impossibilidade de distinguir as operações especulativas das operações de cobertura, e as demonstrações financeiras podem virar o sujeito de manipulação. Duma descrição detalhada da estrutura dos derivados aumenta a transparência da função deles.

Cabe constatar que no âmbito checo, as unidades contabilísticas seriam confrontadas com dificuldades sérias ao cumprirem os requisitos das normas/padrões IAS 39 e IFRS 7 no ramo da publicação das informações sobre os contratos de derivados e estrutura deles.

\subsection{Problemas relacionados com a aplicação facultativa da contabilidade de cobertura}

O maior risco da contabilidade de cobertura consiste em exame da efectividade das relações de cobertura. Além de muitas empresas não conseguirem examinar suas relações de cobertura, muitas receiam, e é um receio justo, que o resultado do respectivo exame pode não corresponder com o intervalo desejado de $\langle-80 \%$; $-125 \%\rangle$.

Até em casos que permitam a aplicação de fair-value option dentro do âmbito checo, parece certo que as empresas aproveitariam apenas a cobertura dos fluxo de caixa. Com respeito a isso expressamos duvidas sobre uma perspectiva promissória das coberturas de valores justos, se a aplicação de „fair-value option“ oferece para as unidades contabilísticas a possibilidade de reavaliar o item coberto (sendo esse um instrumento financeiro) ao valor justo, evitando o dever de satisfazer os requisitos da contabilidade de cobertura.

A maioria das entidades não publica informações mais detalhadas sobre a contabilidade de cobertura. Em resultado, o investidor não recebe informações nem sobre a parte do volume das operações de derivados associado com a cobertura do valor justo, nem sobre a do fluxo de caixa. Quase sempre faltam informações sobre a medição da eficácia das relações de cobertura. Além disso, os leitores externos das demonstrações financeiras raramente entendem, que parte dos valores nominais de cobertura era pouco eficaz e quantas relações de cobertura terminara em consequência de falta da efectividade.

\section{CONCLUSÕES}

Na República Checa, as demonstrações financeiras elaboradas pelas sociedades cujos títulos patrimoniais não são negociáveis publicamente, sofrem da certa influencia dos requisitos correspondentes com as normas/padrões internacionais de IFRS.

No âmbito dos títulos de valores verificou-se, conforme Tabela 1, a semelhança dos requisitos para o reconhecimento inicial em valor de aquisição, e reavaliação consecutiva realizada conforme o grau até que o respectivo instrumento financeiro será enquadrado na respectiva carteira de títulos.

É o ramo dos investimentos em títulos patrimoniais onde é recomendável a adoção das carteiras válidas nas normas internacionais (HFT, AFS), e a aplicação consecutiva dos requisitos idênticos com os das normas. Ao contrário, não foi encontrada muita compatibilidade das normas checas com os requisitos da IFRS no reconhecimento dos instrumentos de dívida.

Verificou-se, sobretudo em caso dos instrumentos de dívida de longo prazo, uma solução inconveniente (apesar de elegante, visto do ponto de vista fiscal) da amortização da diferença entre o valor nominal e o preço de emissão das obrigações. Assim seria conveniente introduzir a carteira de HTM no âmbito checo, sob condição de terem sido resolvidas as consequências fiscais, i.e. do ponto de vista do investidor, a amortização dos prémios vai exercer efeito fiscal no instrumento de dívida. 
Tabela 1 - Comparação Análise Base a Jaccard Coefficients

\begin{tabular}{|c|c|c|c|}
\hline \multirow{2}{*}{\multicolumn{2}{|c|}{ Comparação }} & \multicolumn{2}{|c|}{ CZE/IFRS } \\
\hline & & $\mathrm{S}_{\mathrm{ij}}$ & $\mathrm{D}_{\mathrm{ij}}$ \\
\hline \multicolumn{4}{|c|}{ Financial assets } \\
\hline & Recognition & 1.000 & 0.000 \\
\hline & Initial measurement & 1.000 & 0.000 \\
\hline & Subsequent measurement & 1.000 & 0.000 \\
\hline & Disclosure & 0.000 & 1.000 \\
\hline & Derecognition & 0.000 & 1.000 \\
\hline \multicolumn{4}{|c|}{ Financial liabilities } \\
\hline & Recognition & 1.000 & 0.000 \\
\hline & Initial measurement & 1.000 & 0.000 \\
\hline & Subsequent measurement & 0.500 & 0.500 \\
\hline & Disclosure & 0.000 & 1.000 \\
\hline & Derecognition & 1.000 & 0.000 \\
\hline \multicolumn{4}{|c|}{ Equity instruments } \\
\hline & Recognition & 0.000 & 1.000 \\
\hline & Purchase of own shares & 0.667 & 0.333 \\
\hline & Dividends on ordinary shares & 1.000 & 0.000 \\
\hline \multicolumn{4}{|l|}{ Derivatives } \\
\hline & Initial measurement & 0.000 & 1.000 \\
\hline & Subsequent measurement & 1.000 & 0.000 \\
\hline \multicolumn{4}{|c|}{ Hedge accounting } \\
\hline & Recognition & 1.000 & 0.000 \\
\hline & Fair value hedges measurement & 1.000 & 0.000 \\
\hline & Cash flow hedges measurement & 1.000 & 0.000 \\
\hline & $\begin{array}{l}\text { Hedging of net investments in foreign operations } \\
\text { measurement }\end{array}$ & 1.000 & 0.000 \\
\hline & Disclosure & 0.667 & 0.333 \\
\hline \multicolumn{2}{|l|}{ Total } & 0.692 & 0.308 \\
\hline
\end{tabular}

$\mathrm{S}_{\mathrm{ij}}$ - semelhança

$\mathrm{D}_{\mathrm{ij}}$ - dessemelhança

O reconhecimento contabilístico dos contratos de derivados cumpre os requisitos de IFRS. Permanece uma distribuição insuficiente das informações e dos dados sobre as empresas que negociam os contratos de derivados, e também faltam informações públicas sobre esses contratos.

A contabilidade de cobertura tinha ganhado popularidade na República Checa. A motivação principal consiste no impacto fiscal, visto que a Lei sobre o imposto de renda dispunha que os custo dos derivados contratados com o objectivo de cobrar eram dedutíveis. As dúvidas sobre o conceito de cobertura - económico ou contabilístico - eram sujeitos de muitas discussões. Graças a essa disposição, as empresas começaram a aplicar o conceito da contabilidade de cobertura.

O futuro da contabilidade de cobertura na República Checa depende, até certo ponto, do início da aplicação geral de fair-value option no âmbito checo. As unidades contabilísticas da República Checa preferem uma reavaliação dessas relações de cobertura por meio do capital, e em resultado, cobertura de fluxos de caixa.

O maior problema da contabilidade de cobertura consiste na sua implementação prática pelas empresas, sendo essas muitas vezes incapazes de elaborar a documentação de cobertura, e ainda menos de medir a efectividade das suas operações de cobertura. Isso provavelmente causa a pouca vontade das empresas publicarem informações sobre suas estratégias e relações de cobertura. 


\section{REFERÊNCIAS}

AISBITT, Sally. Measurement of harmony of financial reporting within and between countries: the case of the nordic countries. European Accounting Review, v. 10, n. 1, p. 22$51,2001$.

ALEXANDER, David; NOBES, Christopher. Financial accounting: an international introduction. New York: Editora Prentice Hall, 2004.

ASHBAUGH, Hollis. Non-U.S. firms ' accounting standards choices. Journal of Accounting and Public Policy, v. 20, n. 2, p. 129-153, 2001. doi:10.1016/S0278-4254(01)00025-4

BARNES, Ronnie. Accounting for derivatives and corporate risk management policies. Working paper, London Business School, 2001.

BARTH, Mary; LANDSMAN, Wayne et al. Fair value accounting: effects on banks earnings volatility, regulatory capital, and value of contractual cash flows. Journal of Banking and Finance, v. 19, p. 577-605, Jun., 1995. doi:10.1016/0378-4266(94)00141-O

BROWN, Philip; TARCA, Ann. A commentary on issues relating to the enforcement of international financial reporting standards in the EU. European Accounting Review, v. 14, n. 1, p. 181-212, 2005. doi:10.1080/0963818042000338997

COOKE, T. The impact of size, stock market listing and industry type on disclosure in the annual reports of Japanese listed corporations. Accounting and Business Research, v. 22, n. 87, p. 229-237, 1992.

DUMONTIER, Pascal; RAFFOURNIER, Bernard. Why firms comply voluntary with IAS: an empirical analysis with Swiss data. Journal of International Financial Management and Accounting, v. 9, n. 3, p. 216-245, 1998. doi:10.1111/1467-646X.00038

EL-GAZZAR, Samir et al. An empirical investigation of multinational firms ' compliance with international accounting standards. International Journal of Accounting, v. 34, n. 2, p. 239-248, 1999.

EMENYONU, Emanuel; GRAY, Sidney. EC accounting harmonisation: an empirical study of measurement practice in France, Germany and the UK. Accounting and Business Research, v. 23, Winter, p. 49-58, 1992.

EMENYONU, Emanuel; GRAY, Sidney. International accounting harmonisation and the major developed stock market countries: an empirical study. International Journal of Accounting, v. 31, n. 3, p. 269-280, 1996. doi:10.1016/S0020-7063(96)90020-5

HERRMANN, Don; THOMAS, Wayne. Harmonisation of accounting measurement practices in the European Community. Accounting and Business Research, v. 25, n. 100, p. 253-265, 1995.

HUGHES, John et al. Public disclosure of forward contracts and revelation of proprietary information. Working paper, University of California, 2002.

IASB. International Financial Reporting Standards. London: IASB, 2007.

JORGENSEN, Bjorn. Hedging and performance evaluation. Working paper, Northwestern University, 1997.

LEFTWICH, Richard. Evidence of the impact of mandatory changes in accounting principles on Corporate Loan Agreements. Journal of Accounting and Economics, p. 3-36, 1981. 
LEUZ, Christian. IAS versus US GAAP: information asymmetry-based evidence from Germany's New Market. Journal of Accounting Research, v. 41, n. 3, p. 445-472, 2003.

LEUZ, Christian; VERRECCHIA, Robert. The economic consequences of increased disclosure. Journal of Accounting Research, v. 38, Supplement, p. 91-124, 2000.

MCANALLY, Mary. Banks, risk and FASB 105 disclosures. Working paper, University of Texas, Austin, 1995.

MEEK, Gary et al. Factors influencing voluntary annual report disclosures by U.S., U.K. and Continental European multinational corporations. Journal of International Business Studies, v. 26, n. 3, p. 555-572, 1995. doi:10.1057/palgrave.jibs.8490186

MELUMAD, Nahum et al. Comparing alternative hedge accounting standards: shareholders' perspective. Review of Accounting Studies, v. 4, p. 265-292, 1999.

MURPHY, Ann. Firm characteristics of Swiss companies that utilize international accounting standards. International J. of Accounting, v. 35, n. 1, p. 121-131, 1999.

PIRCHEGGER, Barbara. Hedge accounting incentives for cash flow hedges of forecasted transactions. European Accounting Review, v. 15, n. 1, p. 115-135, 2006. doi:10.1080/09638180500510509

RIFFE, S. The valuation of off-balance sheet financial instruments disclosures in the banking industry. Working paper, Southern Methodist University, Dallas, 1993.

WHITTINGTON, Geoffrey. The adoption of international accounting standards in the European Union. European Accounting Review, v. 14, n. 1, p. 127-153, 2005. doi:10.1080/0963818042000338022 